\title{
Algunos poliquetos holoplanctónicos (Annelida: Polychaeta) del Parque Nacional Isla del Coco, Costa Rica
}

\author{
Soledad Jiménez-Cueto ${ }^{1}$, Eduardo Suárez-Morales ${ }^{2}$ \& Álvaro Morales-Ramírez ${ }^{3}$ \\ 1. Biouniverzoo, Chetumal, Quintana Roo 77010, México; soledadjimenezc@hotmail.com \\ 2. El Colegio de la Frontera Sur (ECOSUR), Unidad Chetumal, Av. Centenario Km 5.5, Chetumal, Quintana Roo 77000 , \\ México; esuarez@ecosur.mx \\ 3. Centro de Investigación en Ciencias del Mar y Limnología CIMAR, y Escuela de Biología, Universidad de Costa \\ Rica, San José, Costa Rica; alvaro.morales@ucr.ac.cr
}

Recibido 01-III-2012. Corregido 11-VI-2012. Aceptado 17-IX-2012.

\begin{abstract}
Some holoplanktonic polychaetes (Annelida: Polychaeta) from the Parque Nacional Isla del Coco, Costa Rica. The holoplanktonic polychaetes have not been surveyed in the oceanic waters adjacent to Isla del Coco. These pelagic forms are studied based on the analysis of zooplankton samples collected in the area. Samples were obtained both at night and daytime by standard plankton nets $(0.2-0.5 \mathrm{~mm}$ meshes, $0.49 \mathrm{~m}$ mouth diameter) hauled horizontally and vertically off the oceanic island Isla del Coco, Costa Rica in the Eastern Tropical Pacific. Pelagic polychaetes were collected in one of the night samples and were taxonomically analyzed. The material obtained includes representatives of six species and five families: Alciopidae, Lopadorrhynchidae, Tomopteridae, Polynoidae, and Typhloscolecidae. Brief diagnoses, synonymies, comparative morphological comments, taxonomic illustrations, and data on the distribution of the species collected in the plankton of Isla del Coco are provided. The record of Drieschia pellucida Moore is the first of the species in the Eastern Tropical Pacific; our specimen shows some differences with respect to the original description. The rest of the species have been recorded previously in waters of the Eastern Tropical Pacific, but are the first records of this important protected area. Citation: Jiménez-Cueto, S., E. Suárez-Morales \& Á. Morales-Ramírez. 2012. Algunos poliquetos holoplanctónicos (Annelida: Polychaeta) del Parque Nacional de Isla del Coco, Costa Rica. Rev. Biol. Trop. 60 (Suppl. 3): 207-222. Epub 2012 Dec 01.
\end{abstract}

Key words: marine zooplankton, pelagic polychaetes, plankton, marine biodiversity.

Los poliquetos pelágicos son uno de los grupos taxonómicos menos atendidos en los estudios del zooplancton marino. Representan un grupo derivado de formas bénticas que se ha adaptado totalmente a la vida en la columna de agua (Struck \& Halanych 2010). La importancia del estudio de este grupo reside en sus particulares patrones biogeográficos y en sus cualidades como indicadores de masas de agua (Fernández-Álamo \& Thuesen 1999, Fernández-Álamo \& Färber-Lorda 2006).

Entre los trabajos que se han publicado respecto a los poliquetos pelágicos del Océano Pacífico se encuentran los de Berkeley y Berkeley (1960) y Tebble (1962) en la región del
Pacifico norte y la zona de transición subártica. Fernández-Álamo (1983), en el estudio más completo del grupo en el Pacífico Tropical Oriental, determinó un total de 44 especies que corresponden a 20 géneros y seis familias. Particularmente, en aguas oceánicas del Pacífico de Costa Rica, se cuenta con el trabajo de Hernández-Ramírez (1987), quien registró 22 especies, 11 géneros y cinco familias en la zona del Domo de Costa Rica (VicencioAguilar \& Fernández-Álamo 1996). A pesar de los antecedentes regionales y locales, la fauna de poliquetos pelágicos del polígono marino del Parque Nacional Isla del Coco no ha sido explorada previamente (Morales-Ramírez 
2008), aunque ya se cuenta con importante información de los poliquetos bénticos para la Isla (Dean et al. 2012).

En este trabajo se presenta una reseña de los poliquetos holoplanctónicos encontrados a partir del análisis de muestras de zooplancton obtenidas en el área adyacente al Parque Nacional Isla del Coco. Se presentan diagnosis de las especies pertenecientes a cinco familias, tres de ellas consideradas como taxa hermanos dentro de los Phyllodocidae (Struck \& Halanych 2010): Typhloscolecidae, Lopadorhynchidae y Alciopidae. Los Tomopteridae parecen ser un grupo independiente y en el caso de los Polynoidae, se trata de una familia esencialmente béntica con algunos miembros pelágicos (Støp-Bowitz 1992). Se incluyen comentarios comparativos, ilustraciones taxonómicas e información acerca de la distribución de las especies recolectadas en el Parque Nacional Isla del Coco.

\section{MATERIALES Y MÉTODOS}

Durante la última semana de noviembre del 2001 se llevó a cabo el crucero oceanográfico S-177 a bordo del R/V Robert Seamans con la intención de obtener muestras para evaluar distintos aspectos biológicos y geológicos del polígono del Parque Nacional de la Isla del Coco en aguas oceánicas del Pacífico Tropical Oriental (Graziano 2001; Cortés 2008). Se realizaron muestreos de zooplancton alrededor de la isla, en seis estaciones que representaron distintos ambientes: Bahía Chatham, Bahía Wafer, Wafer externo, Punta María, Isla Dos Amigos, Bahía Yglesias y Cabo Atrevido (Fig. 1). Los arrastres de plancton fueron verticales $(0-50 \mathrm{~m})$ y horizontales; se usaron redes de plancton con $0.49 \mathrm{~m}$ de diámetro de boca y mallas filtrantes de 200 y $500 \mu \mathrm{m}$ en muestreos realizados de día y de noche. Cada red se equipó con un flujómetro Hydrobios para determinar el volumen

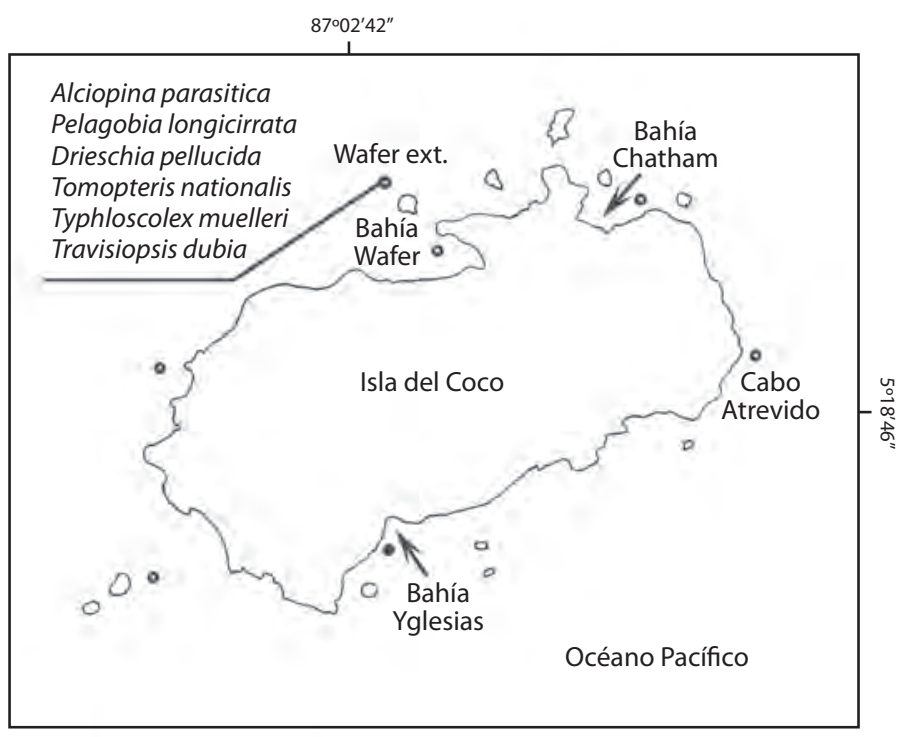

Fig. 1. Localización de las estaciones de muestreo de zooplancton en la zona aledaña al Parque Nacional Isla del Coco, Costa Rica; se muestra la estación Wafer externa, en la que fueron recolectados los poliquetos holoplanctónicos.

Fig. 1. Location of zooplankton sampling stations in the adjacent zone of the Isla del Coco National Park, Costa Rica; the Wafer external station, where the holoplanktonic polychaetes were collected, is shown. 
de agua filtrado. Las muestras fueron fijadas en formaldehído al $4 \%$ y posteriormente preservadas en etanol al 70\%. Los poliquetos aquí analizados se encontraron solamente en una de las muestras del sitio Wafer externo, que fue obtenida mediante arrastre nocturno $(20: 00 \mathrm{~h})$ vertical (0-40m) en Noviembre 5, 2001 con una red de $0.28 \mathrm{~mm}$ de malla filtrante. Los especímenes así obtenidos fueron identificados siguiendo las claves e ilustraciones en los trabajos de Moore (1903), Dales (1957), Støp-Bowitz (1986, 1992) y Fernández-Álamo (1983). Los ejemplares se encuentran depositados y catalogados en la colección de zooplancton de El Colegio de la Frontera Sur (ECOSUR) en Chetumal, México (ECO-CHZ).

\section{RESULTADOS}

\section{Arreglo Sistemático}

Clase Polychaeta Grube, 1850

Orden Phyllodocida Dales, 1962

Familia Alciopidae Ehlers, 1864

Género Alciopina Claparède \& Panceri, 1867 Alciopina parasitica Claparède \& Panceri, 1867

Familia Lopadorrhynchidae Claparède, 1868

Género Pelagobia Greeff, 1897

Pelagobia longicirrata Gravier, 1911

Familia Polynoidae Malmgren, 1867

Género Drieschia Michaelsen, 1892

Drieschia pellucida Moore, 1903

Familia Tomopteridae Grube, 1848

Género Tomopteris Eschscholtz, 1895

Tomopteris nationalis Apstein, 1900

Familia Typhloscolecidae Uljanin, 1878

Género Typhloscolex Busch, 1851

Typhloscolex muelleri Busch, 1851

Género Travisiopsis Levinsen, 1885

Travisiopsis dubia Støp-Bowitz, 1948

\section{Familia Alciopidae Ehlers, 1864}

Diagnosis: Cuerpo delgado, cilíndrico, transparente (blanquecino en material preservado) y de longitud variable. Ojos voluminosos con un cristalino globular. Faringe eversible con papilas marginales o tentáculos laterales largos. Con 3-5 cirros parapodiales alargados o foliáceos.

\section{Alciopina Claparède \& Panceri, 1867}

Diagnosis: Cuerpo corto, se adelgaza hacia la parte posterior. Prostomio proyectado por delante de los ojos, cinco antenas, un par dorsal, un par ventral y una antena mediodorsal reducida a una protuberancia. Faringe corta con papilas marginales. Cinco pares de cirros tentaculares. Cirros dorsales y ventrales foliáceos. Lóbulos setígeros sin apéndices cirriformes. Setas capilares simples muy delgadas y setas aciculares principalmente en segmentos anteriores. Papilas genitales bien desarrolladas sobre parápodos 9-18.

\section{Alciopina parasitica Claparède \& Panceri, 1867}

(Figs. 2A-D)

Alciopina panceri Buchholz 1869:95. Corynocephalus albomaculatus Fauvel 1916:68, Wesenberg-Lund 1939:36, Zei 1956:53, Rullier, 1965:872, Dales \& Peter 1972:71.

Corynocephalus gazellae Apstein 1893:148. Alciopina parasitica Dales 1957:128, Day 1967:177, Dales \& Peter 1972:71, Uschakov 1972:195, Orensanz \& Ramírez 1973:35, StøpBowitz 1981:479, 1992:54-55, Fernández-Álamo \& Thuesen 1999:599.

Material examinado: 3 ejemplares, 2 q y $1 \hat{0}$, preservados en alcohol, sin disecar, Wafer extrerna (Fig. 1), Isla del Coco, Costa Rica (ECO-CHZ-007558).

Diagnosis: Cuerpo corto y blanquecino. Especímenes recolectados de 4-6 $\mathrm{mm}$ de largo por $0.5 \mathrm{~mm}$ de ancho, en dos ejemplares completos con 24-29 parápodos. Prostomio redondeado. Antenas dorsales y ventrales, robustas, cortas, casi del mismo tamaño, antena mediodorsal representada por una protuberancia medial (flecha en Fig. 2D). Ojos dirigidos lateralmente. Cirros tentaculares digitiformes distribuidos en los tres primeros segmentos, 


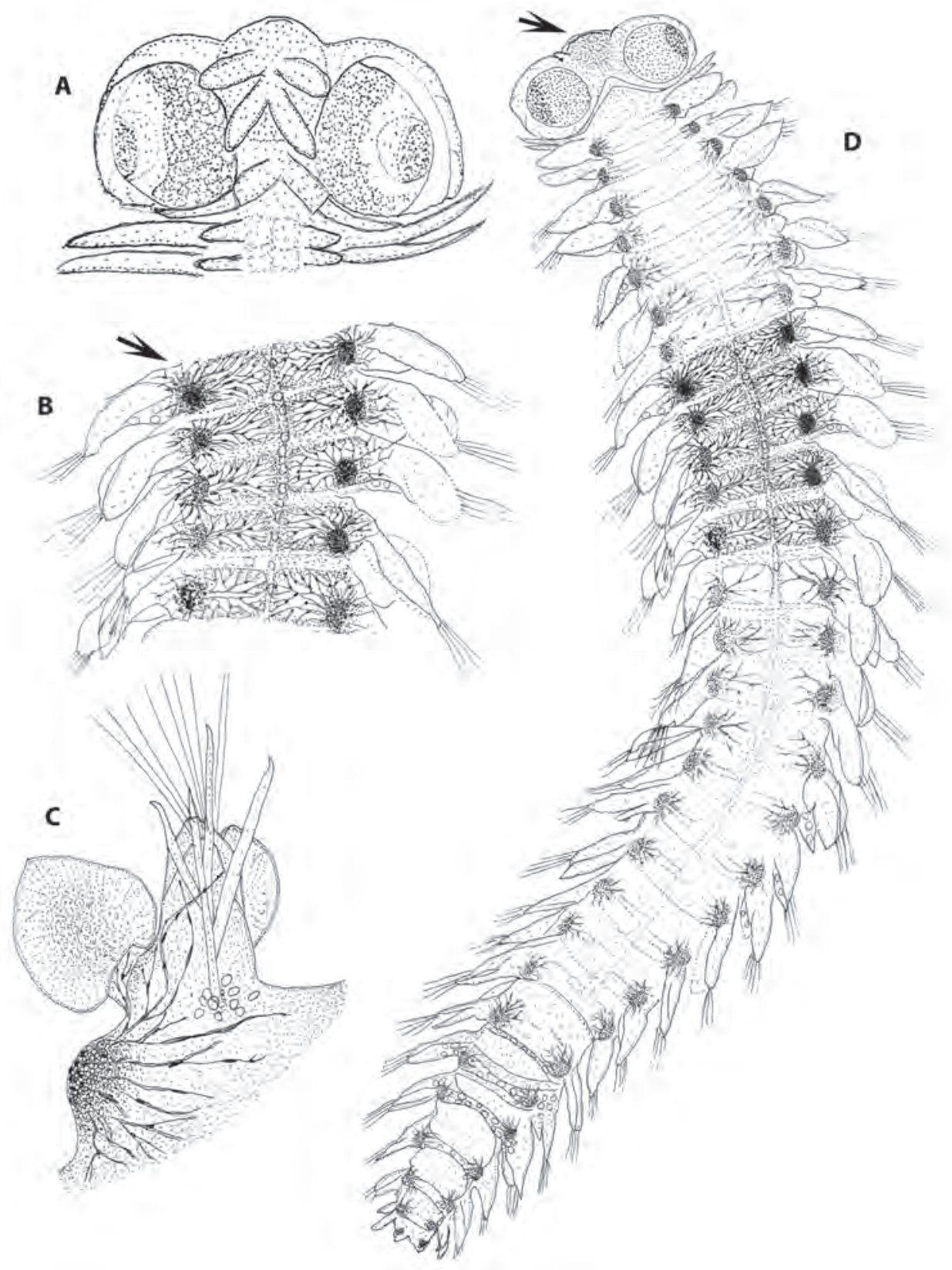

Fig. 2. Alciopina parasitica Claparède \& Panceri, 1867, hembra. Isla del Coco, Costa Rica. (A) prostomio, vista ventral. (B) glándulas segmentales (indicadas por flecha) en parápodos 8-12. (C) parápodo 7, mostrando estructuras parapodiales y glándula segmentaria. (D) espécimen completo, vista dorsal; flecha indica protuberancia de antena media.

Fig. 2. Alciopina parasitica Claparède \& Panceri, 1867, female. Isla del Coco, Costa Rica. (A) prostomium, ventral view. (B) segmentary glands (arrowed) of parapodia 8-12. (C) parapodium 7, showing parapodial structures and segmentary gland. (D) habitus, dorsal view, protuberance of medial antenna arrowed. 
dos pares dorsales largos y anchos y tres pares ventrales, el primero, sobre un pequeño cirróforo aplanado y un tercio más corto que los dorsales, los siguientes dos pares un sexto más cortos que los dorsales. Parápodos con cirros dorsales y ventrales foliáceos, los dorsales son de mayor tamaño. Primer par de parápodos sin lóbulo setígero. Setas aciculares simples, los primeros 13 setígeros presentan 3 ó 4 disminuyendo a 1 en los siguientes setígeros. Setas capilares simples, presentes desde el setígero 4, siendo más abundantes a partir del setígero 14. Glándulas segmentarias ramificadas desde el primer setígero, en los setígeros 8-12 son muy ramificadas apareciendo como bandas sobre todo el setígero. Los machos presentan papilas genitales ventrales en la base de los setígeros 9-13.

Localidad tipo: Golfo de Nápoles (Italia).

Distribución. Day (1975) la considera como una especie marcadamente oceánica y Treadwell (1943) la menciona como una de las especies más comunes en el Océano Pacífico. Ocurre en aguas tropicales del Atlántico Norte y Sur, Mediterráneo y Pacífico Tropical Oriental, Mar Caribe Occidental (Fernández-Álamo 1983, Jiménez-Cueto \& Suárez-Morales 2008). En aguas del Domo de Costa Rica ha sido encontrada por Hernández-Ramírez (1987).

\section{Familia Lopadorrhynchidae Claparède, 1868}

Los Lopadorrhynchidae contienen 15 especies y cuatro géneros, Lopadorrhynchus Grube, 1850, Pelagobia Greeff, 1879, Pedinosoma Reibisch, 1895 y Maupasia Viguier, 1886 (Støp-Bowitz 1986, 1992). Su posición entre los Phyllodocidae ha sido aceptada pero suele ser tratada como un grupo separado (Wilson, 2000). Su biología es poco conocida aunque es posible que sean depredadores en la columna de agua (Uschakov, 1972).

Diagnosis: Cuerpo aplanado dorsoventralmente, prostomio ancho, con cuatro antenas, sin palpos. Ojos débilmente desarrollados. Proboscis eversible, simple. Tres cirros tentaculares en los segmentos 1 y 2 ; pueden tener o no setas. Parápodos unirrámeos con cirros ventrales y dorsales. Setas compuestas con aserraciones distales que son variables y tienen valor taxonómico (Pleijel \& Dales 1991). Pigidio sin cirros.

\section{Pelagobia Greeff, 1879}

Diagnosis: Prostomio con 2 pares de antenas cortas digitiformes y un par de ojos. Dos pares de cirros tentaculares largos y algunas setas cortas. Primer par de parápodos sin cirros dorsales. El resto de los parápodos con cirros dorsales y ventrales alargados y lóbulos setígeros cónicos. Pigidio con dos cirros anales. Faringe lisa con numerosas glándulas pequeñas. Un par de pequeñas mandíbulas.

Pelagobia longicirrata Greeff, 1879

(Figs. 3A-C)

Pelagobia longicirrata Greeff 1879:247, Viguier 1886:377, Reibisch 1895:21, 1905:3, Bergström, 1914:186, Fauvel 1916:61, 1953:131, Benham 1921:57, 1927: 78., Pelagobia viguieri Gravier 1911:311, Chamberlin 1919:122-124. Treadwell 1943:33, Pelagobia erinensis Nolte 1938:278.

Material examinado: $1 \uparrow$ completa, Wafer extrerna, Isla del Coco, Costa Rica (Fig. 1); preservada en alcohol 70\% (ECO-CHZ-07559).

Diagnosis: Organismos con cuerpo deprimido, blanquecinos. La talla del espécimen examinado es de $6 \mathrm{~mm}$ con 17 parápodos. Prostomio cilíndrico, cuatro antenas dorsales, antenas ventrales un tercio más cortas que las dorsales. Lateralmente al prostomio están los órganos nucales que son casi triangulares. Cirros tentaculares anchos y digitiformes, el par dorsal ancho y más largo que el par ventral cuyos cirros son un tercio más cortos. A la altura de estos cirros hay un par de lóbulos setígeros cortos con un haz de setas pequeñas. Parápodos con cirros dorsales y ventrales largos, lóbulos setígeros aplanados y un poco más largos que el ancho del cuerpo con setas 

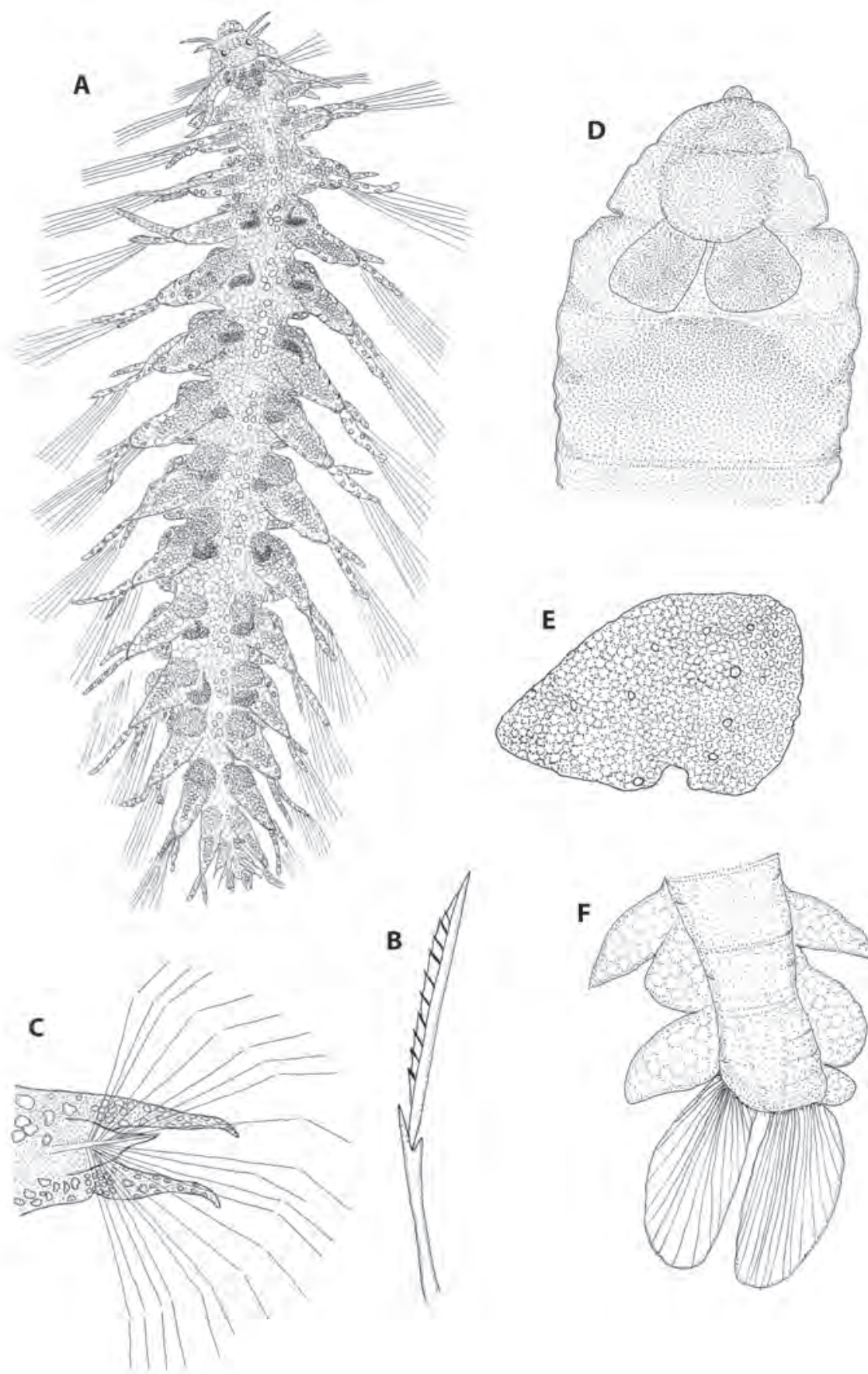

Fig. 3. Pelagobia longicirrata Greeff, 1879, Isla del Coco, Costa Rica, hembra. (A) espécimen completo, vista dorsal. (B) seta, parápodo 6. (C) parápodo 6. Travisiopsis dubia Støp-Bowitz, 1948. (D) parte anterior, vista ventral. (E) cirro parapodial. (F) parte posterior del cuerpo, vista ventral.

Fig. 3. Pelagobia longicirrata Greeff, 1879, Isla del Coco, Costa Rica, female. (A) complete specimen, dorsal view. (B) seta, setigerous segment 6. (C) parapodium 6. Travisiopsis dubia Støp-Bowitz, 1948. (D) anterior part, ventral view. (E) parapodial cirrus. (F) posterior part of body, ventral view. 
capilares compuestas con articulación basal lisa; se extienden formando un abanico.

Comentarios: Especie frecuente en las muestras de zooplancton, considerada como cosmopolita (Støp-Bowitz, 1992). Esta especie puede medir hasta $12 \mathrm{~mm}$ de longitud por $2.0 \mathrm{~mm}$ de ancho con un máximo de 25 setígeros; las medidas de nuestro espécimen lo ubican como un ejemplar mediano.

Localidad tipo: Atlántico Oriental, Islas Canarias.

Distribución: De acuerdo con Dales (1957) esta es la especie más frecuente y abundante de los Phyllodocida pelágicos, su distribución alcanza latitudes frías y tropicales. En el Pacífico tropical de México fue registrada como la forma oceánica más abundante (Fernández-Álamo \& Sanvicente-Añorve 2005). Se encuentra en el Atlántico, Mediterráneo, Pacífico Norte y Noroeste. En aguas de Costa Rica ha sido registrada por Dean (2009) y en el Domo de Costa Rica por Hernández-Ramírez (1987).

\section{Familia Polynoidae Malmgren, 1867}

Diagnosis: Cuerpo dorsoventralmente aplanado. Con tres antenas y regularmente con 7-21 pares de élitros insertados a partir de segundo segmentos. Se alternan con cirros dorsales (Pettibone 1982). Los polinoidos son esencialmente formas bénticas, sin embargo, sus formas larvarias pueden ser encontradas en el plancton. Varias especies de los géneros Drieschia, Drieschella y Herdmanella son consideradas verdaderamente pelágicas (StøpBowitz 1992, 1996).

\section{Drieschia Michaelsen, 1892}

Diagnosis: Antenas con inserción terminal, parápodos sesquiremes (o casi), con acículas dorsales y ventrales, pero sin setas dorsales. Cirros tentaculares sin setas en su base; dos tipos de setas ventrales. Número de setígeros: 25-30, con 12 a 14 pares de élitros. Actualmente el género contiene cinco especies nominales: D. elegans Seidler, 1924, D. melanostoma Ditlevsen, 1917, D. nans (Chamberlin, 1919),
D. pelagica Michaelsen, 1892 y D. pellucida Moore, 1903.

Drieschia pellucida Moore, 1903

(Figs. 4A-H)

Drieschia pellucida Moore, 1903:794, Seidler 1923:174.

Drieschia atlantica Treadwell 1936:52.

Material examinado: 1 , espécimen incompleto con 21 segmentos, Wafer externa, Isla del Coco, Costa Rica (Fig. 1) (ECO-CHZ-07557).

Diagnosis: Prostomio sin procesos frontales; dos pares de ojos poco conspicuos en arreglo trapezoidal. Antena media dos veces más larga que las laterales (esta característica no fue observada en el ejemplar de Isla del Coco, estas estructuras están ausentes). Dos palpos acuminados. Cirros tentaculares con una pequeña protuberancia cerca de la base y casi del mismo tamaño que los palpos. Parápodos sesquiremes, notopodios reducidos a un cirro dorsal con acícula y sin setas; neuropodio redondeado. Ejemplar examinado con 11 pares de élitros evidenciados por la presencia de elitróforos distribuidos en los segmentos 2, 4, $5,7,9,11,13,15,17,19$ y 21 alternados con cirros dorsales en cirróforos dorsales voluminosos, cilíndricos. Los cirros y cirróforos son de mayor tamaño que los elitróforos. Haz de setas con diferentes tipos de setas, las superiores de la rama ventral son numerosas, largas y delgadas; el eje cilíndrico se abulta ligeramente hacia la parte distal, que termina en una punta afilada. Las setas ventrales inferiores, que son 3-7, son más cortas y gruesas, ambas espinosas unidentadas, su parte distal es corta.

Comentarios: El individuo encontrado en las aguas aledañas a Isla del Coco tiene una longitud total de $6 \mathrm{~mm}$. Fue identificado como D. pellucida por poseer las características presentadas en la diagnosis y que se apegan en lo general a la descripción original de Moore (1903). Nuestro espécimen fue comparado también con D. nans (Chamberlin, 1919), una especie cercana que ha sido ubicada como 


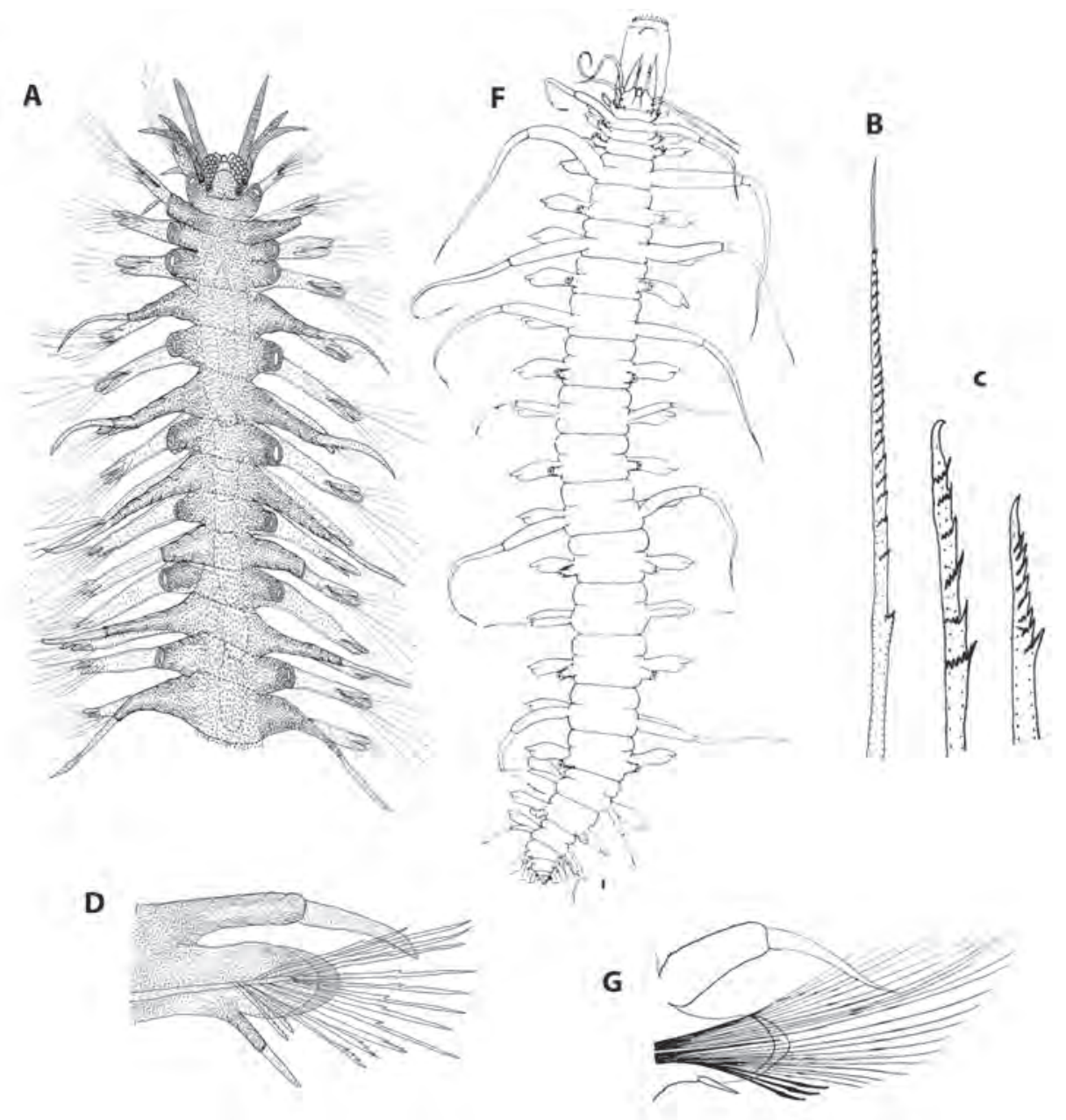

$\mathbf{E}$

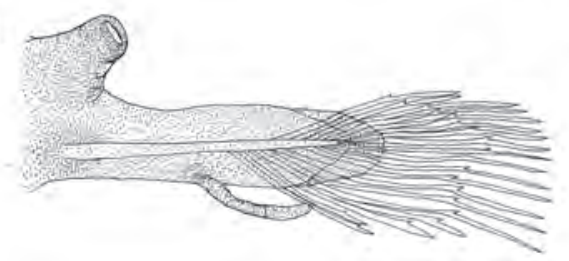

H

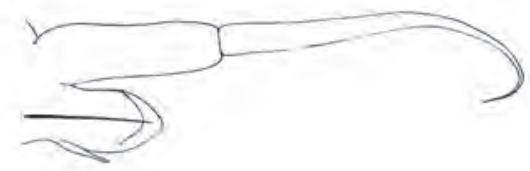

Fig. 4. Drieschia pellucida Moore, 1903, Isla del Coco, Costa Rica. (A) espécimen completo, vista dorsal, ejemplar sin antenas media y lateral. (B) seta larga. (C) parte terminal de dos tipos de setas cortas. (D) parápodo 5. (E) parápodo 14. (F) holotipo completo (ilustración original de Moore 1903), vista dorsal. (G) parápodo 10 del holotipo. (H) parápodo 14 del holotipo.

Fig. 4. Drieschia pellucida Moore, 1903, Isla del Coco, Costa Rica. (A) complete specimen, dorsal view. (B) long chaeta. (C) terminal part of two kinds of short chaetae. (D) parapodium 5. (E) parapodium 14. (F) holotype specimen (original illustration from Moore 1903), dorsal view. (G) parapodium 10 of holotype. (H) parapodium 14 of holotype. 
sinónimo de D. pellucida (Støp-Bowitz 1992, 1996) pero que actualmente es considerada válida (Read \& Fauchald 2011). El individuo recolectado en Isla del Coco difiere de esta especie debido a que carece de un cirróforo modificado en el quinto segmento, característico de D. nans (Chamberlin 1919, Rozbaczylo et al. 2004). Además, el primer cirro es mucho más largo que las setas, mientras que en nuestro ejemplar es aproximadamente del mismo tamaño. En D. pelagica las setas son muy delgadas y flexibles (Michaelsen 1892 figs. 16, 17), no rígidas como en $D$. pellucida y las setas robustas son curvas. Nuestro ejemplar difiere también de $D$. melanostoma, otra especie similar, porque en ésta la parte distal de las setas pequeñas y robustas es bífida (Ditlevsen 1917, fig. 21), mientras que en $D$. pellucida es simple (Fig. 4C). Sin embargo, nuestro especimen difiere en algunos aspectos respecto a la descripción original: los cirros tentaculares fueron descritos originalmente (Moore 1903) como estructuras de mayor tamaño que los palpos, mientras que en el espécimen de Isla del Coco el tamaño de ambas estructuras es similar. En el holotipo (Moore 1903) los cirróforos de los parápodos 14 y 18 son de mayor tamaño que el lóbulo setígero (Fig. 4H), mientras que en nuestro espécimen el lóbulo setígero es considerablemente menor que el cirróforo (Fig. 4D). Adicionalmente, en el holotipo el cirro sobrepasa el haz de setas mientras que no ocurre así en nuestro ejemplar. A pesar de que son variaciones importantes respecto a la descripción original y al igual que en esta se trata de sólo un individuo, consideramos que se le debe reconocer por ahora como D. pellucida. Se espera la obtención de más especímenes de la región para evaluar si estas diferencias son consistentes y pudieran revelar una especie indescrita.

Localidad tipo: Atlántico occidental, costas de Massachusetts, Estados Unidos.

Distribución: Se ha registrado en el Atlántico occidental (Bermudas, costa oriental de Norteamérica) y oriental (frente a las costas de Guinea). En el Pacífico sólo se ha registrado en las cercanías de Isla de Pascua (Støp-Bowitz 1992; Rozbaczylo et al. 2004).
Comentario: Su hallazgo representa el primer registro de esta especie en aguas de Costa Rica y del Pacífico Tropical Oriental.

\section{Familia Tomopteridae Johnston, 1865 Tomopteris Eschscholtz, 1825}

Prostomio fusionado con los primeros dos segmentos. Con un par de antenas divergentes, un par de ojos débilmente desarrollados y dos órganos nucales. Primer segmento libre, con un par de apéndices reducidos o ausentes en la etapa adulta. Segundo segmento libre, con un par de apéndices más largos que el cuerpo. Proboscis corta, sin papilas u otras ornamentaciones. Parápodos birrámeos, sin setas. En algunas especies los últimos parápodos se reducen para formar una cola. Gónadas siempre en las ramas dorsales, en ocasiones también en las ventrales. Ramas parapodiales bordeadas por pínulas membranosas con distintos tipos de glándulas como las cromófilas, sólo en pínulas ventrales de segmentos 1-5. Glándulas hialinas en las pínulas dorsales de los segmentos $3 \mathrm{y}$ 4 o en la parte apical de las pínulas ventrales. Glándulas roseta, con centro amarillo o rojizo, bioluminiscentes (Uschakov, 1972) en el tronco parapodial de los dos primeros parápodos o en ambas pínulas parapodiales cerca del ápice.

Tomopteris nationalis Apstein, 1900

(Figs. 5A-D)

Apstein, 1900:41; Dales, 1957:139; Day, 1967:198-204; Dales \& Peter, 1972:74; Day, 1975:210; Fernández-Álamo, 1983:173.

Material examinado: 1 ejemplar adulto, Wafer externa, Isla del Coco, Costa Rica (Fig. 1); espécimen incompleto (ECO-CHZ-07562).

Diagnosis: Talla del ejemplar analizado: $1.2 \mathrm{~mm}$ de largo. Número de parápodos: 12 . Antenas prostomiales gruesas, con una muesca central. Primer apéndice cirriforme igual de largo o algo más largo que la antena. Segundo apéndice cirriforme del mismo largo del cuerpo o más largo. Prostomio con un par de ojos lenticulados. Parápodos robustos y largos, 

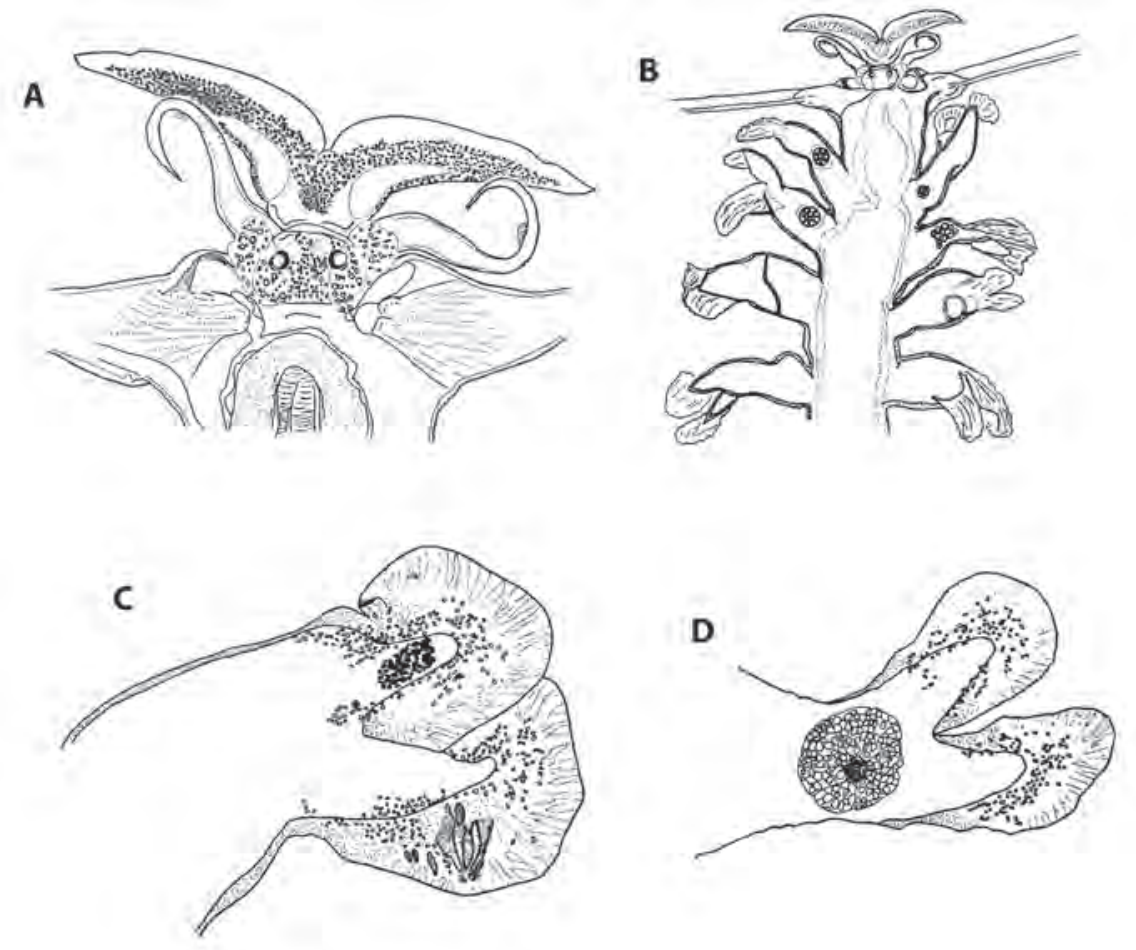

Fig. 5. Tomopteris nationalis Apstein, 1900, Isla del Coco, Costa Rica. (A) parte anterior, vista dorsal. (B) detalle de la parte anterior. (C) parápodo 1. (D) parápodo 5.

Fig. 5. Tomopteris nationalis Apstein, 1900, Isla del Coco, Costa Rica. (A) anterior region, dorsal view. (B) detail of anterior region. (C) parapodium 1. (D) parapodium 5.

más anchos que el cuerpo. Glándulas en roseta presentes en el tronco de los parápodos 1 y 2 . Glándulas cromófilas desde el tercer al último parápodos. Gónadas en algunos parápodos.

Localidad tipo: Nápoles (Italia).

Distribución: Aguas tropicales del Atlántico y Pacífico (Fernández-Álamo 1983, StøpBowitz 1986, Jiménez-Cueto \& Suárez-Morales 1999, Díaz et al. 2009). Se ha registrado en aguas del Pacífico Tropical Oriental (Fernández-Álamo 2000) y en el Domo de Costa Rica (Hernández-Ramírez 1987, Vicencio-Aguilar \& Fernández-Álamo 1996). Esta especie suele aparecer en aguas neríticas (Jiménez-Cueto \& Suárez-Morales 1999).

\section{Familia Typhloscolecidae Uljanin, 1878}

Diagnosis: Cuerpo fusiforme o cilíndrico, translúcido o blanquecino. Prostomio cónico con un pálpodo digitiforme, sin ojos, frecuentemente con carúncula y un par de órganos nucales bien diferenciados, órgano en retorta en la pared dorsal de la faringe. Los tres primeros segmentos con un par de cirros tentaculares foliáceos dirigidos hacia adelante envolviendo el extremo anterior. A partir del segmento cuatro presentan cirros dorsales y ventrales dehiscentes. Parápodos con lóbulos setígeros rudimentarios, con pocas setas aciculares. Pigidio con un par de cirros anales aplanados. 
Comentarios: Los Typhloscolecidae son un pequeño grupo de tres géneros. Se conoce poco acerca de su biología pero se ha propuesto, con base en su morfología, que son ectoparásitos de zoopláncteres gelatinosos (Øresland \& Pleijel 1991).

Typhloscolex Busch, 1851

Diagnosis: Cuerpo delgado, cilíndrico y transparente. Prostomio redondeado con un pálpodo digitiforme. Órganos nucales bien diferenciados en la parte dorsal. Dos grandes lóbulos ciliados dorsal y ventralmente. La proboscis es inerme, el órgano en retorta, ciliado, que puede salir parcial o totalmente por la boca cuando está extendida. Extremo anterior rodeado por los cirros foliáceos de los tres primeros segmentos. Setas presentes a partir del segmento 5. Pigidio con cirros anales. Se conocen 6 especies; la especie tipo es T. muelleri Busch, 1851.

Thyphloscolex muelleri Busch, 1851

(Figs. 6A-C)

Typhloscolex mülleri Busch 1851: 115

Sagitella bobretzkii Wagner 1872:347

Sagitella barbata Uljanin 1878:6

Sagitella praecox Uljanin 1878:8

Acicularia virchowii Greeff 1879:237

Sagitella kowalewskii Wagner 1872:346

Typhloscolex mülleri Reibisch 1895:52, StøpBowitz 1949:17

Typhloscolex muelleri Day 1967:208, Dales \& Peter 1972:80, Orensanz \& Ramírez 1973:50, Støp-Bowitz 1981:481, 1992:94-95, 1996:181, Fernández-Álamo 1983:187-191，2004:648, Fernández-Álamo \& Thuesen 1999:618.

Material examinado: 2 $q$, Wafer externa, Isla del Coco, Costa Rica (Fig. 1), especímenes completos (ECO-CHZ-07560).

Diagnosis: Prostomio con un pequeño palpo asimétrico inserto en una estructura basal. Lóbulos preorales dorsales y ventrales ciliados. Tres primeros pares de parápodos con un par de cirros parapodiales foliáceos que rodean el extremo distal. Lóbulos setígeros cónicos y pequeños en los primeros cinco segmentos. Pigidio con cirros anales ovalados que pueden ser o no ciliados. Ejemplares examinados con 25 parápodos $(3.1-3.5 \mathrm{~mm})$, hasta el setígero 15 con 3 setas aciculares, los últimos 10 con 2 setas solamente. Cirros anales no ciliados.

Comentarios: La talla de los especímenes del Pacífico Tropical Oriental y de latitudes tropicales y subtropicales varía entre $1.0 \mathrm{a}$ $7.5 \mathrm{~mm}$, con un promedio de $2.7 \mathrm{~mm}$ (Fernández-Álamo 2004). Por tanto, los especímenes de Isla del Coco son de talla promedio.

Localidad tipo: Trieste, Mediterráneo.

Distribución: Day (1975) la registra como una especie primordialmente oceánica que puede encontrarse también en aguas neríticas. Atlántico, Mediterráneo, Pacífico Norte y Noroeste; Pacífico Tropical Oriental, incluyendo aguas oceánicas cercanas a la Isla del Coco (ver Fernández-Álamo 1983, 2004). En aguas del Domo de Costa Rica ha sido encontrada por Hernández-Ramírez (1987) y Vicencio-Aguilar \& Fernández-Álamo (1996).

Travisiopsis Levinsen, 1885

Diagnosis: Cuerpo alargado, poco traslúcido. Prostomio cónico con un pálpodo, generalmente con carúncula en la parte dorsal. Órganos nucales libres. Primeros tres segmentos con cirros foliáceos rodeando la parte anterior del prostomio. Siguientes segmentos con cirros dorsales y ventrales. Lóbulos setígeros pequeños, con pocas setas. Pigidio con dos cirros anales ovalados. Seis especies conocidas; la especie tipo es T. lobifera Levinsen, 1885.

Travisiopsis dubia Støp-Bowitz, 1948 (Figs. 3D-F)

Travisiopsis dubia Dales 1960:485; Tebble 1962:414-415; Day 1967:212-213; Dales \& Peter 1972:82; Orensanz \& Ramírez 1973:54; Fernández-Álamo 1983:197. 


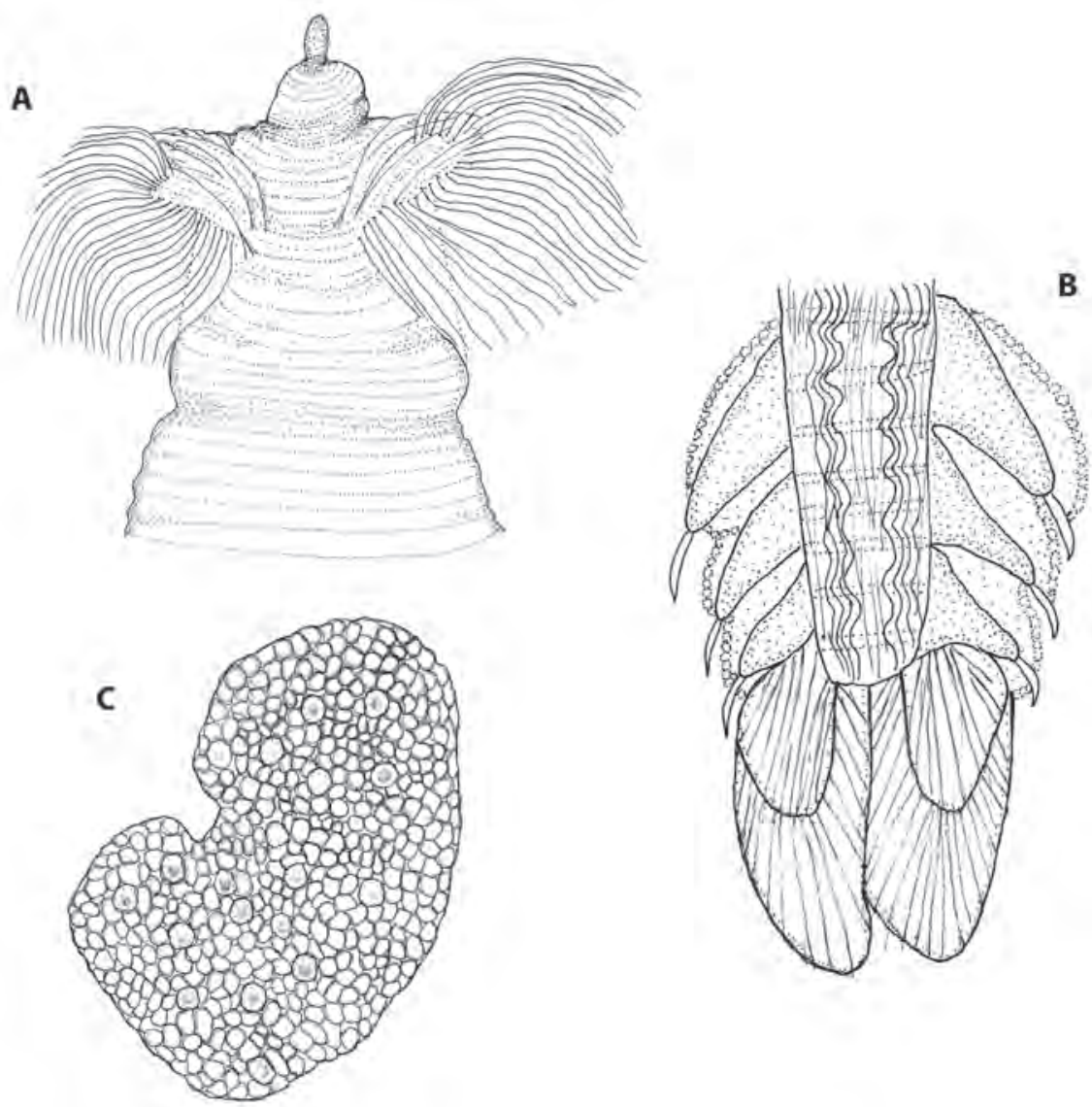

Fig. 6. Typhloscolex muelleri Busch, Isla del Coco, Costa Rica. (A) extremo anterior, vista ventral. (B) extremo anterior, vista ventral, otro individuo. (C) cirro parapodial.

Fig. 6. Typhloscolex muelleri Busch, Isla del Coco, Costa Rica. (A) anterior end, ventral view. (B) anterior end, ventral view, another specimen. (C) parapodial cirrus.

Material examinado: $1+$, ejemplar completo, Wafer externa, Isla del Coco, Costa Rica (Fig. 1) (ECO-CHZ-07561).

Diagnosis: Especie pequeña $(6-10 \mathrm{~mm}$, 28 segmentos; individuo de Isla del Coco: $5.8 \mathrm{~mm})$, de cuerpo alargado; región cefálica con tres segmentos, cada uno con un par de cirros lamelares y cubren la parte frontal del prostomio en vista lateral. En el segundo y tercer segmentos los cirros son ovales. Cuerpo con 19-25 segmentos con cirros dorsales y ventrales. Prostomio cónico, protuberante dorsalmente con un palpo anterior. Lóbulos nucales presentes, redondeados. Setas aciculares simples, pequeñas, en grupos de tres. Cirros anales alargados y curvos distalmente.

Localidad tipo: Atlántico Norte.

Distribución: Atlántico Norte, Pacífico Norte, Océano Índico. Ocurre en el Pacífico Tropical (Fernández-Álamo 1991, SalazarVallejo \& Londoño-Mesa 2004). Existen sólo unos pocos registros confirmados de esta especie en aguas del Pacífico (Fernández-Álamo 1991, 2004). En aguas del Domo de Costa Rica ha sido encontrada por Hernández-Ramírez (1987) (Vicencio-Aguilar \& Fernández-Álamo 1996). De acuerdo con los datos de Day (1975), esta especie puede ser igualmente abundante en 
aguas oceánicas, de plataforma y costeras, por lo que su presencia cerca de la isla del Coco era esperable.

\section{DISCUSIÓN}

En general, el estudio de los poliquetos pelágicos ha sido relegado a un plano marginal cuando se realizan estudios faunísticos de estos invertebrados, esto puede estar relacionado con su diversidad relativamente baja y desde luego, con la diferencia en las técnicas de muestreo para estudiar las formas del plancton y las bénticas. A manera de comparación, Dean et al. (2012) establecieron 103 nuevos registros de poliquetos bénticos para la Isla y comentan acerca de sus afinidades taxonómicas con las especies de las Islas Galápagos.

En su reciente revisión acerca de la diversidad de los poliquetos de Costa Rica, Dean (2009) menciona solamente algunos registros de especies pelágicas: Lopadorhynchus nationalis Reibisch, Pelagobia longicirrata, Rhynconereella moebii (Apstein), Tomopteris kefersteini Greeff y un registro dudoso de $T$. nisseni. No se incluyen los registros de 23 especies encontradas en aguas del Domo de Costa Rica (ver Vicencio-Aguilar \& Fernández-Álamo 1996).

Los poliquetos pelágicos de la isla sólo han sido estudiados marginalmente; el único registro previo de una especie pelágica en las cercanías de Isla del Coco fue en el trabajo de Treadwell (1928), quien reconoció la presencia del tomoptérido Tomopteris nisseni Rosa, 1908 (Dean et al. 2012). Por ello, este es el primer análisis enfocado a este grupo de poliquetos en Isla del Coco y las especies aquí encontradas representan los primeros registros para esta área marina protegida. Existen varios registros en la zona oceánica aledaña como es el Domo de Costa Rica (ver Vicencio-Aguilar \& Fernández-Álamo 1996) y en otras zonas del Pacífico Tropical Oriental (Fernández-Álamo 1983), que en suma incluyen más de 40 especies. Por ello, es notable que el hallazgo de estas seis especies se haya generado a partir de un muestreo puntual y limitado. Las formas pelágicas son poco comunes en aguas nerítico-costeras (Suárez-Morales et al. 2005, Díaz et al. 2009), por lo que se espera que nuevos muestreos oceánicos en los alrededores de la Isla del Coco generarán registros adicionales de estas formas ampliamente distribuidas en aguas tropicales del Pacífico. También se deben considerar muestreos de aguas profundas $(>200 \mathrm{~m})$, pues muchas especies realizan amplias migraciones verticales o habitan aguas mesopelágicas; estas muestras suelen tener una diversidad importante de poliquetos holoplanctónicos (ver Rozbaczylo et al. 2004).

En general, se espera que la fauna de poliquetos de Isla del Coco sea muy similar a la del Pacífico Tropical Oriental y en particular a la del Domo de Costa Rica. Con la excepción de Drieschia pellucida, todas las especies registradas en la zona aledaña a Isla del Coco se han encontrado en el Domo de Costa Rica y áreas cercanas del Pacífico Tropical (FernándezÁlamo 1983, Vicencio-Aguilar \& FernándezÁlamo 1996).

El registro de D. pellucida es interesante debido a que no se ha encontrado previamente en aguas del Pacífico Tropical Oriental; se trata de una especie conocida sólo en el Atlántico (Støp-Bowitz 1986, 1992). Støp-Bowitz (1992) considera a D. nans (Chamberlin, 1919) como un sinónimo de D. pellucida pero Fauchald (2011) la considera válida. Drieschia nans sólo ha sido registrada en aguas del Pacífico Suroriental (Chamberlin 1919); Rozbaczylo et al. (2004) encontraron sólo un individuo en las cercanías de la Isla de Pascua e Isla San Félix, en aguas chilenas. Se considera una especie restringida a las aguas templadas de la región sudoriental del Pacífico, por lo que su registro en aguas tropicales resulta improbable. Ello parece apoyar la idea de que se trata de dos especies distintas, por lo que la aparición de $D$. pellucida en el área de estudio, con caracteres que difieren de la descripción original representa un hallazgo interesante también desde el punto de vista taxonómico, por lo que deben analizarse más ejemplares para determinar el estatus, variabilidad y la distribución real de esta especie y de $D$. nans. 


\section{AGRADECIMIENTOS}

Nuestro agradecimiento a todas las personas, técnicos, personal de buque de investigación y colegas que en alguna u otra forma aportaron su tiempo y esfuerzo en el trabajo de campo y de laboratorio en los muestreos realizados durante la expedición oceanográfica a Isla del Coco. Agradecemos a Sergio SalazarVallejo (ECOSUR-Chetumal) sus comentarios respecto a algunas especies incluidas en este estudio y el facilitarnos literatura poco accesible. Rosa Ma. Hernández, a cargo de la colección de zooplancton de ECOSUR, depositó y catalogó los especímenes examinados. Agradecemos los comentarios y sugerencias de dos revisores anónimos cuya aportación permitió mejorar considerablemente una versión previa de este escrito.

\section{RESUMEN}

Los poliquetos holoplanctónicos no han sido estudiados en las aguas oceánicas adyacentes al Parque Isla del Coco. Se estudiaron estas formas pelágicas a partir del análisis de muestras de zooplancton recolectadas en esta área protegida. Las muestras fueron obtenidas mediante redes de plancton estándar $(0.2-0.5 \mathrm{~mm}$ de malla, $0.49 \mathrm{~m}$ de diámetro de boca) en arrastres horizontales y verticales en la isla oceánica Isla del Coco, Costa Rica, en el Pacífico Tropical Oriental. Los poliquetos pelágicos de esta zona fueron analizados taxonómicamente. El material obtenido incluye representantes de seis especies y cinco familias: Alciopidae, Lopadorhynchidae, Tomopteridae, Polynoidae y Typhloscolecidae. Se presentan diagnosis breves, sinonimias, análisis morfológicos comparativos, ilustraciones taxonómicas y la distribución conocida de las especies recolectadas en el plancton de Isla del Coco. El registro de Drieschia pellucida Moore es el primero en el Pacífico Tropical Oriental; nuestro espécimen muestra algunas diferencias respecto a la descripción original. El resto de las especies han sido encontradas previamente en aguas del Pacífico Tropical Oriental y en el Domo de Costa Rica, pero son los primeros registros para esta área protegida.

Palabras clave: zooplancton marino, poliquetos pelágicos, plancton, biodiversidad marina.

\section{REFERENCIAS}

Apstein, C. 1893. Die Alciopiden der Berliner Zoologischer Sammlung. Arch. Natur. 59: 141-150.
Apstein, C. 1900. Die Alciopiden und Tomopteriden der Plankton-Expedition. Ergebn. der Plankton-Exped. der Humboldt-Stiftung 2: 1-61.

Benham, W.B. 1921. Polychaeta. Australian Antarctic Expedition, 1911-14. Sci. Rep., Ser. C, Zool. Bot. 6: 1-128.

Bergström, E. 1914. Zur Systematik des Polychaeten familie der Phyllodociden. Zool. Bidr. Uppsala 3: 37-224.

Berkeley, E. \& C. Berkeley. 1960. Notes on some Polychaeta from the west coast of México, Panamá and California. Can. J. Zool. 38: 357-362.

Busch, W. 1851. Beobachtungen liber Anatomie und Entwicklung einiger wirbellosen Seethiere. Hirschwald, Berlin.

Buchholz, R.W. 1869. Zur Entwicklungsgeschichte von Alciope. Z. Wiss. Zool. 19: 95-98.

Chamberlin, R.V. 1919. The Annelida Polychaeta of the Albatross Tropical Pacific Expedition, 1891-1905. Mem. Mus. Comp. Zool. Harvard College 48: 1-514.

Cortés, J. 2008. Historia de la investigación marina de la Isla del Coco, Costa Rica. Rev. Biol. Trop. 56 (Supl. 2): 1-18.

Dales, R.P. 1957. Pelagic polychaetes of the Pacific Ocean. Bull. Scripps Inst. Oceanogr. 7: 99-168.

Dales, R.P. 1960. Pelagic polychaetes from the Malacca Straits and south China Sea. Ann. Mag. Nat. Hist. 13: 481-487.

Dales, R.P. \& G. Peter. 1972. A synopsis of the pelagic Polychaeta. J. Nat. Hist. 6: 55-92.

Day, J.H. 1967. A monograph on the Polychaeta of Southern Africa. Brit. Mus. Nat. Hist. Publ. 656: 1-878.

Day, J.H. 1975. Zooplancton de la région de Nosy-be X. The biology of planktonic Polychaeta near Nosy-be, Madagascar. Cah. ORSTOM, Ser. Óceanogr. 13: 197-216.

Dean, H. K. 2009. Polychaetes and Echiurans. P. 181-191. In: Wehrtmann, I.S. \& Cortés, J. (eds.) Marine Diversity of Costa Rica, Central America. Monographiae Biologicae. Springer Science + Business Media B. V.

Dean, H.K., J.A. Sibaja-Cordero \& J. Cortés. 2012. Polychaetes (Annelida: Polychaeta) of Coco Island National Park, Pacific Costa Rica. Pac. Sci. 66: en prensa.

Díaz, O.F., E. Radha-Henríquez, I. Liñero-Arana \& B. Marín. 2009. Poliquetos holoplanctónicos (Annelida: Polychaeta) de la plataforma del Atlántico venezolano. Ciencia y Mar 13: 15-26.

Ditlevsen, H. 1917. Annelids. Danish-Ingolf Exped. 4: $1-71$.

Fauvel, P. 1916. Annélides polychètes pélagiques prevenant des campagnes de l'Hirondelle et la Princesse Alice (1885-1910). Result. Camp. Sci. Monaco 48: $1-152$. 
Fauvel, P. 1953. The Fauna of India including Pakistan, Ceylon, Burma and Malaya. Annelida Polychaeta. Indian Press, Allahabad, India.

Fernández-Álamo, M.A. 1983. Los poliquetos pelágicos (Annelida: Polychaeta) del Pacífico Tropical Oriental: Sistemática y Zoogeografía. Tesis Doctoral. Facultad de Ciencias, UNAM, México.

Fernández-Álamo, M.A. 1991. Holoplanktonic polychaetes from the Gulf of California: August-September 1977. CalCOFI Rep. 32: 97-104.

Fernández-Álamo, M.A. 2000. Tomopterids (Annelida: Polychaeta) from the Eastern Tropical Pacific Ocean. Bull. Mar. Sci. 67:45-53

Fernández-Álamo, M.A. 2004. Distribution of the holoplanktonic typhloscolecids (Annelida: Polychaeta) in the eastern Tropical Pacific Ocean. J Plankton Res. 26: 647-657.

Fernández-Álamo, M.A. \& J. Färber-Lorda. 2006. Zooplankton and the oceanography of the Eastern Tropical Pacific: a review. Prog. Oceanogr. 69: 318-359.

Fernández-Álamo, M.A. \& L. Sanvicente-Añorve. 2005. Holoplanktonic polychaetes from the Gulf of Tehuantepec, Mexico. Cah. Biol. Mar. 46: 227-239.

Fernández-Álamo, M.A. \& E. Thuesen. 1999. Polychaeta, p. 595-619. In: Boltovskoy, D. (ed.) South Atlantic Zooplankton. Vol. 1. Backhuys Publ. Leiden.

Gravier, C. 1911. Espéces nouvelles d'Annélides polychètes. Expédition Antarctique Française du "Pourquoi-Pas", dirigee par le Dr. J.B. Charcot (1908-1910). Bull. Mus. Hist. Nat. Paris 17: 310-316.

Graziano, L. 2001. Cruise Report S-177, Scientific Activities Undertaken Aboard SSV Robert C. Seamans, San Diego, California-Puntarenas, Costa Rica, October 10 2001-November 18, 2001. Sea Education Association, Woods Hole, Massachusetts. 45p.

Greeff, R. 1879. Ueber pelagische Anneliden von der Küste der Canarischen Inseln. Z. wiss. Zool., 32: 237-284.

Hartman, O. 1969. Atlas of the Sedentariate Polychaetous Annelids from California. Allan Hancock Foundation, University of Southern California, Los Angeles.

Hernández- Ramírez, M.B. 1987. Distribución de los poliquetos pelágicos (Annelida: Polychaeta) en la región de surgencias denominada "Domo de Costa Rica" durante los periodos Febrero-Marzo de 1979 y Julio-Agosto de 1982. Tesis Profesional. Facultad de Ciencias, UNAM.

Jiménez-Cueto, S. \& E. Suárez-Morales. 1999. Tomopterids (Polychaeta: Tomopteridae) of the western Caribbean Sea. Bull. Inst. Royal Scien. Nat. Belgique, Biol. 69: 5-14.

Jiménez-Cueto, S. \& E. Suárez-Morales. 2008. An account of Alciopina, Torrea, and Rhynconereella
(Polychaeta: Alciopidae) of the western Caribbean Sea. Belg. J. Zool. 138: 70-80.

Michaelsen, W. 1892. Polychaeten von Ceylon. Jahr. Hamb. Wiss. Anst. 9: 1-23.

Moore, J.P. 1903. Some pelagic Polychaeta new to the Woods Hole fauna. Proc. Acad. Nat. Sci. Philadelphia 55: 793-801.

Morales-Ramírez, A. 2008. Caracterización cualitativa del zooplancton del Área de Conservación Marina Isla del Coco (ACMIC), Océano Pacífico de Costa Rica. Rev. Biol. Trop.56 (Supl. 2): 159-169.

Nolte, W. 1938. Annelidenlarven. Nordisches Plankton, Ser. Lieferung, 24: 171-282.

Orensanz, J. \& F. Ramírez. 1973. Taxonomía y distribución de los poliquetos pelágicos del Atlántico Sudoccidental. Bol. Inst. Biol. Mar del Plata 21: 1-122.

Øresland, V. \& F. Pleijel. 1991. An ectoparasitic typhloscolecid polychaete on the chaetognath Eukrohnia hamata from the Antarctic Peninsula. Mar. Biol. 108: 429-432.

Pettibone, M.H.1982.Annelida, p. 1-43. In S.P. Parker (ed.). Synopsis and classification of living organism, McGraw-Hill, Nueva York.

Pleijel, F. \& R.P. Dales. 1991. Polychaetes: British Phyllodocoideans, Typhloscolecoideans and Tomopteroideans. Synop. British Fauna, New Ser. 45: 1-202.

Read, G. \& K. Fauchald. 2011. World Polychaeta database at http://www.marinespecies.org/polychaeta/aphia. php? $\mathrm{p}=$ taxdetails\&id=332773 (Consultado: 2 febrero 2012).

Reibisch, J.G.P. 1895. Die Pelagischen Phyllodociden und Typhloscoleciden der Plankton-Expedition. Ergebn. Plankton-Exped. Humboldt-Stiftung IIc: 1-63.

Rozbaczylo, N., R.A. Moreno, G. Guzmán \& J Jaque. 2004. Poliquetos pelágicos (Annelida, Polychaeta) del Pacífico suroriental frente a Chile e islas oceánicas. Invest. Mar., Valparaíso 32: 11-22.

Rullier, F. 1965. Quelques annélides polychètes pélagiques d'Anno-Bon. Bull. Inst. Fran. Afr. Noire 27: 866-875.

Salazar-Vallejo, S.I. \& M.H. Londoño-Mesa. 2004. Lista de especies y bibliografía de poliquetos (Polychaeta) del Pacífico Oriental Tropical. An. Inst. Biol. Univ. Nac. Autón. Méx., Ser. Zool. 75: 9-97.

Seidler, H.J. 1923. Beiträge zur Kenntnis der Polynoiden I. Arch. Natur. Ab. A, 89: 1-217.

Støp-Bowitz, C. 1948. Polychaeta from the Michael Sars North Atlantic deep-sea expedition 1910. Rep. Sci. Res. M. Sars North Atlantic Deep-Sea Exp. 5: 1-91.

Støp-Bowitz, C. 1949. Polychètes pélagiques des expéditions norvégiens antarctiques de la Norvegia 19271928, 1928-1929 et 1930-1931. Sci. Res. Norv. Ant. Exped. 31: 1-25. 
Støp-Bowitz, C. 1981. Polychaeta, p. 471-492. In D. Boltovskoy (ed). Atlas del zooplancton del Atlántico sudoccidental. INIDEP, Buenos Aires, Argentina.

Støp-Bowitz, C. 1992. Polychètes pélagiques des campagnes de "l'Ombango" dans les eaux équatoriales et tropicales ouest-africaines (Etudes et Thèses). ORSTOM, Francia.

Støp-Bowitz, C. 1996. Polychaeta, p. 149-189. In R. Gasca \& E. Suárez-Morales (eds). Introducción al estudio del zooplancton marino. El Colegio de la Frontera Sur (ECOSUR), CONACYT, México.

Struck, T.H. \& Halanych, K.M. 2010. Origins of holopelagic Typhloscolecidae and Lopadorhynchidae within Phyllodocidae (Phyllodocida, Annelida). Zool. Scr. 39: 269-275.

Suárez-Morales, E., S. Jiménez-Cueto \& S. Salazar-Vallejo. 2005. Catálogo de los Poliquetos (Polychaeta) pelágicos del Golfo de México y Mar Caribe Mexicano. ECOSUR/ CONACYT-SEMARNAT, México.

Tebble, N. 1962. The distribution of pelagic polychaetes across the North Pacific Ocean. Bull. Br. Mus. Nat. Hist. Zool. 7: 371-492.

Treadwell, A.L. 1928. Polychaetous annelids from the Arcturus Oceanographic Expedition. Zoologica 8: 449-489.

Treadwell, A.L. 1936. Polychaetous annelids from the vicinity of Nonsuch Island, Bermuda. Zoologica 21: 49-68.

Treadwell, A.L. 1943. Biological results of the last cruise of "Carnegie". III. Polychaetous annelids. Carnegie Inst. Washington, D.C., Publ. 555: 30-59.
Uljanin, B. 1878. Sur le genre Sagitella N. Wagner. Arch. Zool. Exp. Gén 7:1-32.

Uschakov, P.B. 1955. Polychaeta of far eastern seas of the USSR. Acad. Sc. SSSR. 56: 1-445.

Uschakov, P.B. 1972. Polychaeta 1. Polychaetes of the suborder Phyllodociforma of the Polar Basin and the northwestern part of the Pacific (translated from Russian by the Israel Program for Scientific Translation, Jerusalem 1974). Fauna SSSR 102: 1-271.

Vicencio-Aguilar, M. \& M.A. Fernández-Álamo. 1996. Zooplancton del domo de Costa Rica: taxonomía y biogeografía. Rev. Biol. Trop. 44: 631-642.

Viguier, C. 1886. Etudes sur les Animaux inferieurs de la Baie d'Alger. Recherches sur les Annelides pélagiques. Arch. Zool. Expér. Gén. 4: 1-347

Wagner, B.N. 1872. Nouveau groupe d'Annélides. Trav. Nat. St. Petersburg 3: 344-347.

Wesenberg-Lund, E. 1939. Pelagic polychaetes of the families Aphroditidae, Phyllodocidae, Lopadorhynchidae and Alciopidae. Report on the Danish Oceanographical Expedition 1908-10 to the Mediterranean and adjacent seas. Biology 2: 1-46.

Wilson, R.S. 2000. Family Lopadorhynchidae, p. 134136. In P.L. Beesley, G.J.B. Ross \& C.J. Glasby (eds). Polychaetes and Allies: the Southern Synthesis. CSIRO Publ., Melbourne, Australia.

Zei, M. 1956. Pelagic polychaetes of the Adriatic. An ecological study of the geographical and seasonal distribution of the families Tomopteridae, Alciopidae, Phyllodocidae and Typhloscolecidae. Thal. Jugoslav. 1: 33-68. 